\title{
Enlarging Regions of Stable Running with Segmented Legs
}

\author{
Juergen Rummel*, Fumiya Iida ${ }^{\dagger}$, James Andrew Smith* and Andre Seyfarth* \\ ${ }^{*}$ Locomotion Laboratory, Institute of Sports Science, University of Jena, Germany \\ Email: juergen.rummel@uni-jena.de, andre.seyfarth@uni-jena.de, \\ ${ }^{\dagger}$ Robot Locomotion Group, CSAIL, Massachusetts Institute of Technology, Cambridge, MA \\ Email: iida@csail.mit.edu
}

\begin{abstract}
In human and animal running spring-like leg behavior is found, and similar concepts have been demonstrated by various robotic systems in the past. In general, a spring-mass model provides self-stabilizing characteristics against external perturbations originated in leg-ground interactions and motor control. Although most of these systems made use of linear spring-like legs. The question addressed in this paper is the influence of leg segmentation (i.e. the use of rotational joint and two limb-segments) to the self-stability of running, as it appears to be a common design principle in nature. This paper shows that, with the leg segmentation, the system is able to perform self-stable running behavior in significantly broader ranges of running speed and control parameters (e.g. control of angle of attack at touchdown, and adjustment of spring stiffness) by exploiting a nonlinear relationship between leg force and leg compression. The concept is investigated by using a twosegment leg model and a robotic platform, which demonstrate the plausibility in the real world.
\end{abstract}

\section{INTRODUCTION}

With the eventual goal of adaptive legged locomotion systems, self-stabilizing characteristics of underactuated legged robots have been extensively studied since the pioneering work by Raibert [1]. While many legged systems control the leg joints to achieve stable movements, there is another class of control approach to stabilize locomotion behavior by exploiting passive joints.

One of the well-known models, the spring-mass model, also known as Spring-Loaded Inverted Pendulum (SLIP), uses spring-like dynamics for energy-efficient running and walking behaviors [2], [3], [4]. A fascinating characteristic of this model is the self-stability against perturbations such as dynamic leg-ground interactions, and changes of system parameters [5]. A number of studies have previously shown that the concept can be utilized in one-, two-, four- and sixlegged robots [6], [7], [8], [9].

While most of theoretical studies investigated legged models with telescopic joints [10], it is still not fully understood what the role of segmented legs is, i.e. the legs with limbsegments connected through rotational joints, which appears to be a common design principle in nature. More specifically, although many robotic systems intuitively employ segmented legs, e.g. [11], [12], there has been no systematic investigation on the leg segmentation and behavioral performances.

This study was supported by an Emmy-Noether grant of the German Research Foundation (DFG, SE1042).

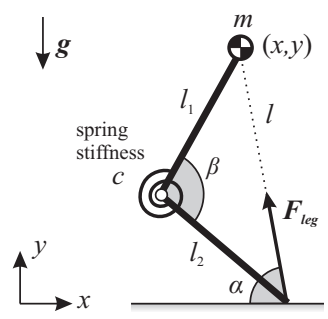

(a)

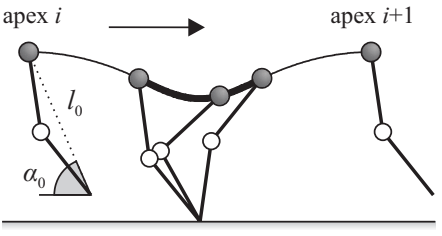

(b)
Fig. 1. (a) The two-segment leg model, and (b) a periodic running sequence. The direction of the intersegmental joint does not influence the running direction.

The goal of this paper is to gain better insights into legged locomotion focusing on segmented legs and their influence on dynamical stability in running. By using a simulation model of two-segment leg with a rotational spring located at the intersegmental joint, and its counterpart robotic platform, we investigate contributions of leg geometry to leg force generation and the resultant running dynamics.

A comparison of the model with the SLIP will help clarifying the role of leg segmentation on running. We expect that the two-segment leg inherits the properties of the SLIP leading to self-stable running with a fixed angle of attack policy. We further assume that the segmented leg shifts regions of self-stability and allows new solutions.

\section{Methods}

\section{A. Leg Templates}

In this study we investigate principles of running using leg templates. The basic template is the spring-mass model [2], [3] that represents the action of the stance leg by a linear spring of constant stiffness $k$ and rest length $l_{0}$. The leg is massless and the body is represented by a point mass. Hence, the leg generated force $F_{l e g}$ can only direct from the fixed foot point to the center of mass. The amount of leg force depends on leg compression $\Delta l(t)=l_{0}-l(t)$ but not on leg orientation or compression velocity. Furthermore, a linear relationship between leg compression and leg force is assumed since the leg stiffness $k$ is constant

$$
F_{l e g}=k \Delta l \text {. }
$$


To investigate the role of segmentation in legged locomotion we propose a two-segment leg template (Fig. 1(a)). In order to keep the analysis simple we take advantage of the above mentioned assumptions made in the spring-mass model: The action of the leg is represented by a leg force depending on leg compression. The segmented leg template is defined by two massless segments of length $l_{1}$ and $l_{2}$ connected by the intersegmental joint with inner angle $\beta$. The spring-like force in a segmented leg is generated by a torsional spring of stiffness $c$ causing a joint torque

$$
\tau(\Delta \beta)=c \Delta \beta
$$

$\Delta \beta$ denotes the joint flexion $\beta_{0}-\beta$ with the rest angle $\beta_{0}$. The joint angle $\beta$ is a function of the leg length $l$

$$
\beta(l)=\arccos \frac{l_{1}^{2}+l_{2}^{2}-l^{2}}{2 l_{1} l_{2}}
$$

To calculate the amount of joint flexion $\Delta \beta$ we need to specify a rest angle $\beta_{0}$ which corresponds to a rest length of the leg

$$
l_{0}\left(\beta_{0}\right)=\sqrt{l_{1}^{2}+l_{2}^{2}-2 l_{1} l_{2} \cos \beta_{0}} .
$$

Thus, any amount of joint flexion $\Delta \beta$ translates into a corresponding leg compression $\Delta l$ depending on the rest angle $\beta_{0}$. The joint torque (2) results in a leg force with

$$
F_{l e g}(\tau)=\frac{l}{l_{1} l_{2}} \frac{\tau}{\sin \beta}
$$

and for any two-segment leg with a rotational spring the corresponding leg force-leg compression dependency $F_{l e g}(\Delta l)$ can be calculated. Compared to the spring-mass model, the two-segment leg has a few more parameters: the segment lengths $l_{1}$ and $l_{2}$, the joint stiffness $c$ and the rest angle $\beta_{0}$. To facilitate the comparison between the two-segment leg and the spring-mass model (parameters $l_{0}$ and $k$ ), we define a reference leg compression at $10 \%$ of leg length $\Delta l_{10 \%}=0.1 l_{0}$. Based on the leg force $F_{10 \%}\left(\Delta l_{10 \%}\right)$, a reference stiffness $k_{10 \%}=F_{10 \%} / \Delta l_{10 \%}$ can be defined. This stiffness can be compared to the leg stiffness of the SLIP.

The equation of motion for both templates is

$$
m \ddot{\boldsymbol{r}}=\boldsymbol{F}_{\boldsymbol{l e g}}+m \boldsymbol{g}
$$

with $\boldsymbol{r}=[x, y]^{T}$ the position of the point mass and $\boldsymbol{g}=[0,-g]^{T}$ the gravitational acceleration. The force vector $\boldsymbol{F}_{\boldsymbol{l e g}}=\left[F_{x}, F_{y}\right]^{T}$ directs from the foot point to the center of mass. Its magnitude is given by (1) and (5) for the springmass model and the two-segment model, respectively. The leg force is zero during flight phase and the center of mass trajectory describes a ballistic curve. We consider transitions between flight and stance by the conditions

$$
\begin{array}{ll}
\text { flight } \Rightarrow \text { stance }: & y(t) \leq l_{0} \sin \alpha_{0} \\
\text { stance } \Rightarrow \text { flight }: & l(t)>l_{0}
\end{array}
$$

where $\alpha_{0}$ is the predefined angle of attack. The system's state is described by the position $\boldsymbol{r}$ and the velocity $\boldsymbol{v}$ of the center of mass. Since the systems are conservative, the horizontal velocity $v_{x}$ during flight depends on the system

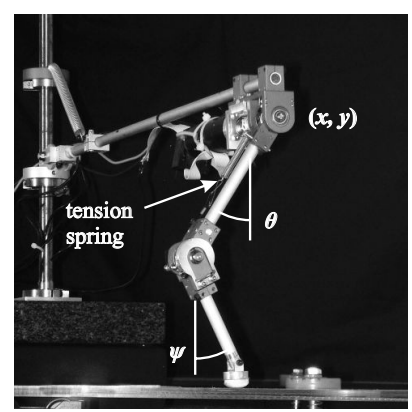

Fig. 2. The single-legged robot testbed JenaHopper and it's four DOF.

energy $E$ and the height at apex $y_{\text {apex }}$ where the vertical velocity $v_{y}$ is alway zero

$$
v_{x}\left(E, y_{\text {apex }}\right)=\sqrt{2 / m\left(E-m g y_{\text {apex }}\right)}
$$

On even ground, the horizontal position $x$ has no influence on the forthcoming stance phase. Hence, for a given energy $E$ the system is fully described by the apex height $y_{\text {apex }}$ if the direction of locomotion does not change. The system energy can be plainly represented by a reference velocity $v_{x}$, ref that appears if the models have an apex height of $y_{\text {apex }}=l_{0}$.

The number of free parameters can be reduced using dimensional analysis [4]. In the spring-mass model three parameters were identified: the dimensionless energy $\widetilde{E}=$ $E /\left(m g l_{0}\right)$, the dimensionless leg stiffness $\widetilde{k}=k l_{0} /(m g)$, and the angle of attack $\widetilde{\alpha}_{0}=\alpha_{0}$. In the two-segment leg we found the torsional stiffness $\widetilde{c}=c /\left(m g l_{0}\right)$, the nominal angle $\widetilde{\beta}_{0}=\beta_{0}$ and the dimensionless segment lengths $\widetilde{l}_{1}=l_{1}$ and $\widetilde{l}_{2}=l_{2}$. In this study, we focus on the case of equal segment lengths $\widetilde{l}_{s}=\widetilde{l}_{1}=\widetilde{l}_{2}$. The dimensionless segment length is then given by $\widetilde{l}_{s}=1 / \sqrt{2\left(1-\cos \widetilde{\beta}_{0}\right)}$. Using this simplification, the two-segment leg has only one more parameter, the nominal angle $\widetilde{\beta}_{0}$, than the spring-mass model. Any combination of $\widetilde{c}$ and $\widetilde{\beta}_{0}$ can be expressed by the dimensionless reference stiffness $\widetilde{k}_{10 \%}=k_{10 \%} l_{0} /(\mathrm{mg})$.

In the simulation study, we use human-like parameters (mass $m=80 \mathrm{~kg}$, leg length $l_{0}=1 \mathrm{~m}$, and earth gravitation $g=9.81 \mathrm{~m} / \mathrm{s}^{2}$.

\section{B. Robot Leg}

The second part of this paper is an experimental study on a robot testbed, called JenaHopper (Fig. 2), which is a singlelegged system with a segmented leg. The intersegmental joint is passive compliant since a tension spring connects both segments. The hip joint is actuated by a DC motor (Sayama RB-35GM) with a 1:100 gear ratio. A supporting boom is attached to the body in order to disable pitch and roll of the body. An elastic material (Adiprene) is attached as foot point. The leg length in nominal position is $l_{0}=0.22 \mathrm{~m}$ and the segment lengths are $l_{1}=0.13 \mathrm{~m}$ and $l_{2}=0.11 \mathrm{~m}$, resulting in a nominal angle of $\beta_{0}=132 \mathrm{deg}$. The total mass is $m=0.42 \mathrm{~kg}$ where the leg is relatively light, accounting for $28 \%$ of $m$. The dimensionless joint stiffness is $\widetilde{c}=0.66$ and the tension spring is somewhat pretensioned. 


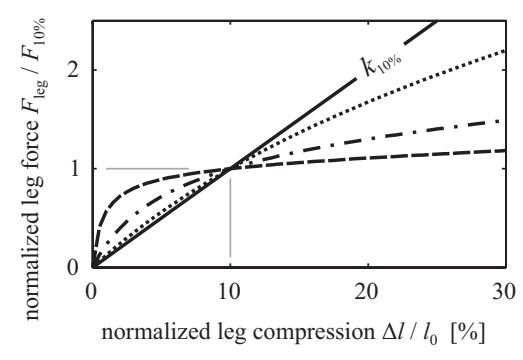

(a)

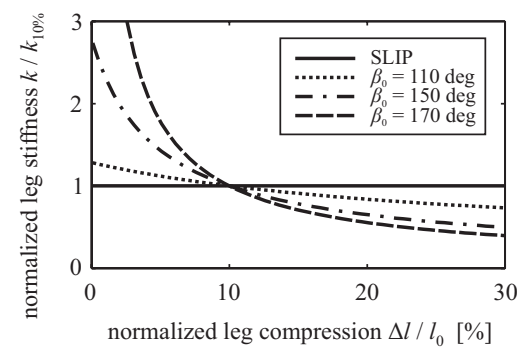

(b)

Fig. 3. (a) Normalized force-length relationships of elastic two-segment legs and, for comparison, the spring-mass model (SLIP) with the same reference stiffness $k_{10 \%}$. The curves in (a) correspond to the normalized stiffness functions in (b).

Since we are interested in the natural behavior of the legged system, we let the leg simply swing back and forth using a sinusoidal oscillation as desired trajectory

$$
\theta_{D}(t)=\theta_{A} \sin (2 \pi f t)+\theta_{0}
$$

with the frequency $f$ and the bias angle $\theta_{0}$. The amplitude $\theta_{A}$ was set such that it decreases with increasing frequency. The amplitude was calculated by $\theta_{A}(f)=80 \mathrm{deg} / \mathrm{s} \cdot f^{-1}-10 \mathrm{deg}$ for $f \geq 2 \mathrm{~Hz}$. For frequencies below $2 \mathrm{~Hz}$ the amplitude was set to $30 \mathrm{deg}$.

During the experiments the robot moved on a turning table. The locomotion patterns were recorded by a Qualisys motion capture system with eight infrared cameras (sampling frequency of $240 \mathrm{~Hz}$ ). The system measured for 30 seconds the trajectories of six tracking points attached at relevant landmarks, e.g. at joints. The joint trajectories were transformed into the saggital plane considering the moving inertial system.

\section{System Analysis}

The dynamic stability of the systems can be analyzed using Poincaré maps of the state vector at a predefined Poincaré section

$$
\boldsymbol{S}_{i+1}=\boldsymbol{F}\left(\boldsymbol{S}_{i}\right)
$$

where $i$ is the number of the individual step and $\boldsymbol{S}_{i}$ denotes the systems state. Limit cycle trajectories or periodic running solutions correspond to a fixed point in the Poincaré map

$$
\boldsymbol{S}^{*}=\boldsymbol{F}\left(\boldsymbol{S}^{*}\right)
$$

The stability of a periodic solution is estimated by calculating the effect of small perturbations away from the fixed point using linear approximation

$$
\left[\boldsymbol{S}_{i+1}-\boldsymbol{S}^{*}\right]=\boldsymbol{J}\left(\boldsymbol{S}^{*}\right)\left[\boldsymbol{S}_{i}-\boldsymbol{S}^{*}\right]
$$

where $\boldsymbol{J}\left(\boldsymbol{S}^{*}\right)$ is the Jacobian matrix and it's eigenvalues are the Floquet multipliers $\lambda_{j}$. If the magnitude of all complexvalued Floquet multipliers is less than one, the limit cycle is stable. In other words, if any $\left|\lambda_{j}\right|$ is greater than one, the periodic solution is unstable.

In this study, the instant of apex during flight phase (Fig. 1(b)) is used as a particular event to formulate the Poincaré map. In this paper, we analyze two kinds of systems, i.e. energy conservative leg template models and a robot. In the former cases (Sec. II-A) the systems are fully described by the apex height $y_{\text {apex }}$ for a given total energy $E$. Therefore, the Poincaré map can be reduced to a one-dimensional map of subsequent apex heights [5].

Investigating experimental data from the robot, the analysis becomes more complicated. The robots state vector $\boldsymbol{S}$ consists of eight elements, i.e. the body position $x$ and $y$, the segment angles $\theta$ and $\psi$, and the velocities of them. During flight phase, the angle of the lower segment $\psi$ depends on $\theta$ since the joint spring was pretensioned and held the joint in its nominal position. Hence, the angle $\psi$ and the angular velocity $\dot{\psi}$ are not necessary in the Poincaré map. The horizontal position $x$ does not influence the forthcoming step, and the vertical velocity $v_{y}$ is always zero at apex. Hence, the Poincaré map is a four-dimensional map of the reduced state vector $\boldsymbol{S}=\left[y, v_{x}, \theta, \dot{\theta}\right]^{T}$.

In experimental recorded data we cannot directly identify a fixed point. Therefore, we define the fixed point $S^{*}$ by the average of the state variables within a trial. The Floquet analysis basically identifies stability of limit cycles for infinite small perturbations which is almost unrealistic for experimental analyses. Here, we define a small perturbation if the apex height $y_{\text {apex }}$ lies within $\pm 0.01 \mathrm{~m}$ from the average $y_{\text {apex }}^{*}$. We are further interested in the systems response if the perturbation is somewhat larger, i.e. if the condition $0.01 \mathrm{~m}<\left|y_{\text {apex }}-y_{\text {apex }}^{*}\right|<0.03 \mathrm{~m}$ is fulfilled.

\section{RESULTS}

\section{A. Leg Mechanics}

Before we examine the effects of leg segmentation on running stability, we first investigate the underlying principles in the compliant two-segment leg. The question here is: How does the leg geometry influence the leg force when the leg becomes compressed? Fig. 3(a) shows the normalized force-length relationship for the two-segment leg at different nominal angles $\beta_{0}$ compared to the linear leg spring model (SLIP). In the segmented leg, the leg force $F_{l e g}$ increases with a high rate of change for small leg compressions. The rate of change decreases the more the leg is flexed. Even for the same reference stiffness $k_{10 \%}$, the force is lower in the two-segment model compared to the linear leg spring model for compressions larger than $10 \%$. The curvature of the force-length relationship further depends on the nominal angle $\beta_{0}$. The more the leg is extended in 

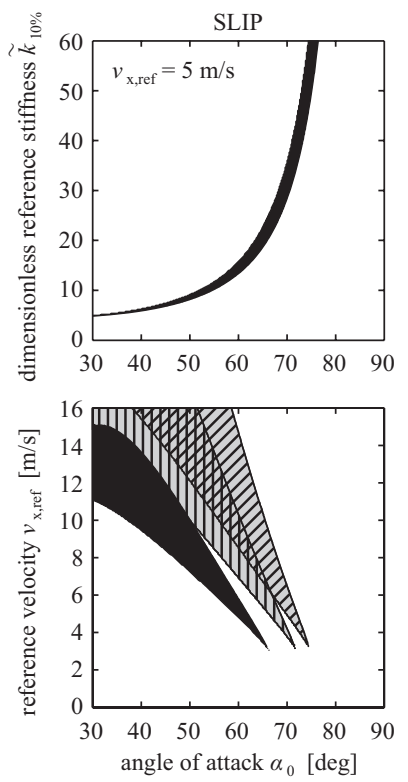

(a)
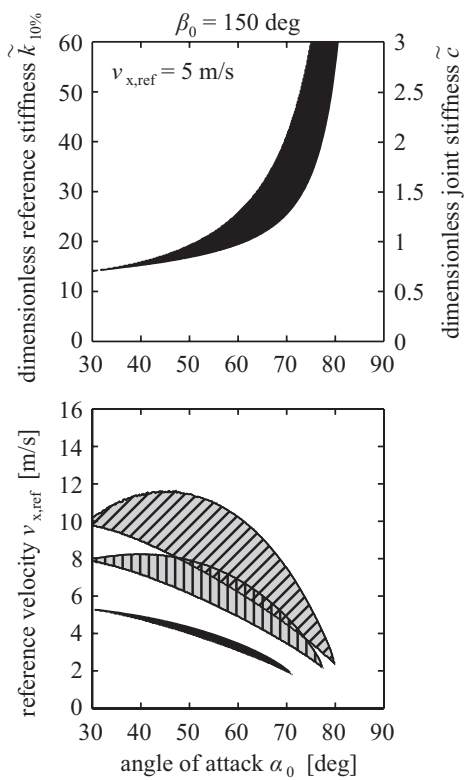

(b)
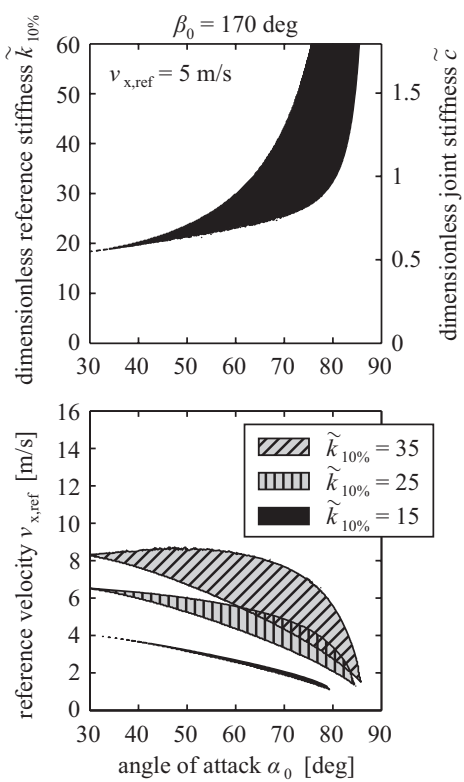

(c)

Fig. 4. Regions of self-stable running for (a) the spring-mass model (SLIP), and (b) and (c) for two configurations of the two-segment leg model. The upper row shows stable system configurations for a given system energy $E$, represented by the reference velocity $v_{x}$,ref. The lower row illustrates regions of stability for some given reference stiffnesses $\widetilde{k}_{10 \%}$. The reference stiffness corresponds to a joint stiffness in the segmented legs as shown on the right axis in (b) and (c).

its nominal position, the more nonlinear becomes the forcelength function. As mentioned in [13], this behavior can be understood as a nonlinear leg stiffness $F_{l e g}(\Delta l) / \Delta l$ while the joint stiffness is constant (Fig. 3(b)).

\section{B. Running Stability}

In this section, we analyze the contribution of leg segmentation on self-stability in running. More precisely, we compare the regions of self-stable running identified in the spring-mass model and the two-segment leg. First, we observe the regions for a given system energy $E$ represented by a moderate reference velocity $v_{x}$, ref of $5 \mathrm{~m} / \mathrm{s}$ (first row in Fig. 4). In the spring-mass model a narrow region of selfstable running (Fig. 4(a)) is found. This region also exists in the segmented leg as shown in Fig. 4(b) and 4(c). In contrast to the linear leg spring model this region is much larger, e.g. for a reference stiffness $\widetilde{k}_{10 \%}$ of 30 the tolerated range in leg angle adjustments $\alpha_{0}$ increases from $2.2 \mathrm{deg}$ in the SLIP to 9.4 and $17.4 \mathrm{deg}$ in the two-segment leg with $\beta_{0}=150$ and $170 \mathrm{deg}$, respectively.

With further increasing stiffness the regions of stable running decrease in the segmented leg. In the template with almost straight leg configuration $\left(\beta_{0}=170 \mathrm{deg}\right)$ the tolerated range in angle of attack diminishes from 17.4 to $9.6 \mathrm{deg}$ for $\vec{k}_{10 \%}=30$ and 60 , respectively.

The regions of self-stability are further shifted to lower speeds in segmented legs as illustrated in the lower row of Fig. 4. The lower limit in reference velocity with stable solutions were found at $v_{x, \text { ref }}=1.9$ and $1.1 \mathrm{~m} / \mathrm{s}$ in the two-segment leg with $\beta_{0}=150$ and $170 \mathrm{deg}$, respectively. Fig. 4(a) shows that in a leg including a linear leg spring

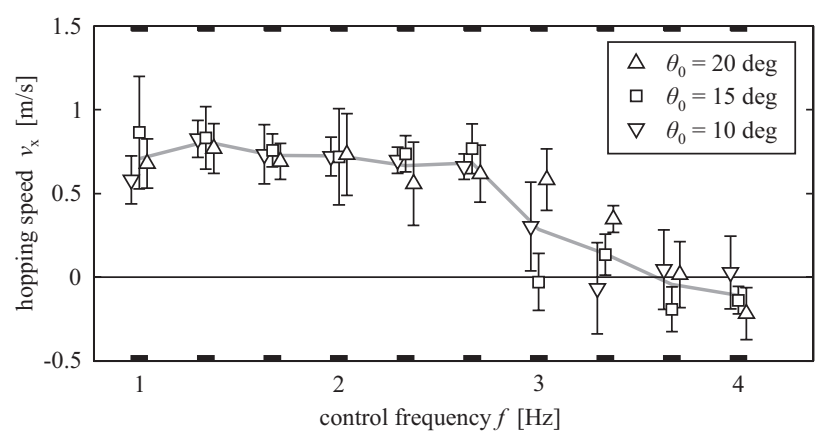

(a)

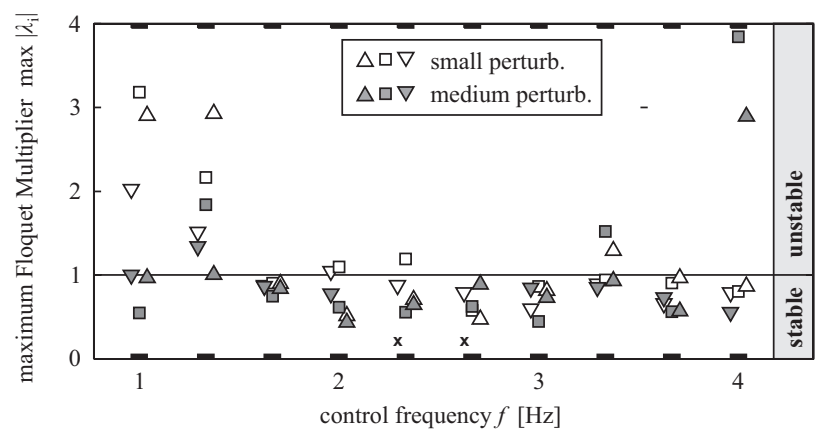

(b)

Fig. 5. (a) Robot speed of each single step (mean \pm s.d.) depending on the oscillation parameters frequency $f$ and offset angle $\theta_{0}$. For each frequency, the symbols are a bit displaced horizontally. The gray line is the grand mean of hopping speed. (b) Floquet multiplier indicating orbital stability of the movement corresponding to the experiments in (a) for small perturbations (empty symbols) and medium perturbations (filled symbols) explained in Sec. II-C. An $\mathbf{x}$ means that no medium or large perturbations occurred. 

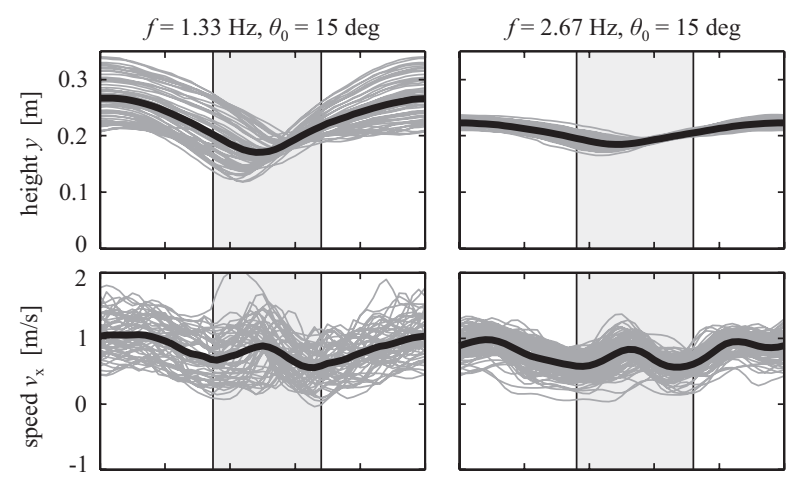

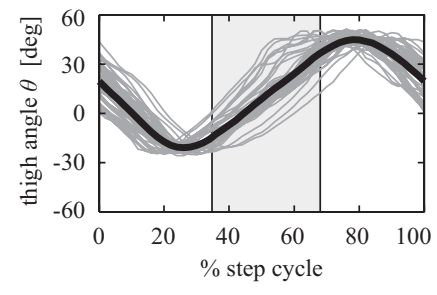

(a)

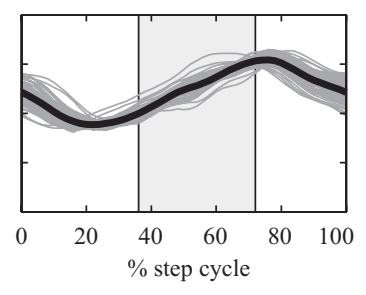

(b)
Fig. 6. Time-series trajectories and the mean traces (thick black line) of robot locomotion for two control frequencies: (a) $f=1.33 \mathrm{~Hz}$, and (b) $f$ $=2.67 \mathrm{~Hz}$. The stance phase is indicated with a gray area. The leg starts retraction (increasing thigh angle) before touch-down.

self-stability disappears below $v_{x, \text { ref }}=3.5 \mathrm{~m} / \mathrm{s}$.

In segmented legs a maximum running speed is found for each configuration that is much lower than in the SLIP. Here, the speed is restricted by the angle of attack. In contrast, the two-segment templates predict the maximum velocity at moderate leg angles $\alpha_{0}$. However, the maximum speed can be shifted to higher values by increasing the joint stiffness.

\section{Robot Experiments}

So far, we have investigated underlying mechanisms and running stability in highly reduced leg templates. In this section we present experimental observed behavior of the two-segment leg robot JenaHopper.

The implemented feed-forward control strategy basically swings the leg back and forth and the robot starts hopping immediately. Fig. 5(a) shows the achieved hopping speeds dependent on the free parameter combinations where hopping was observed. The average speed hardly changes for the range of frequencies from 1.0 to $2.67 \mathrm{~Hz}$. Here, the mean values of the speed lie between 0.6 and $0.9 \mathrm{~m} / \mathrm{s}$. Above $3 \mathrm{~Hz}$ the robot behavior changes from forward locomotion to hopping in place and hopping backwards with further increasing frequency. Fig. 5(b) is a visualization of the orbital stability of the robot movement by means of the Floquet multipliers. Hopping is stable for frequencies between 1.67 and $3.0 \mathrm{~Hz}$, where the Floquet multiplier for small and medium perturbations are almost always lower than one. Unstable locomotion is indicated for lower frequencies $(f<$ $1.5 \mathrm{~Hz}$ ) especially for small perturbations, however, the robot continued hopping. Here, the range where the state variables were measured is relatively large as shown in Fig. 6(a). In the case of hopping in place and hopping backwards with

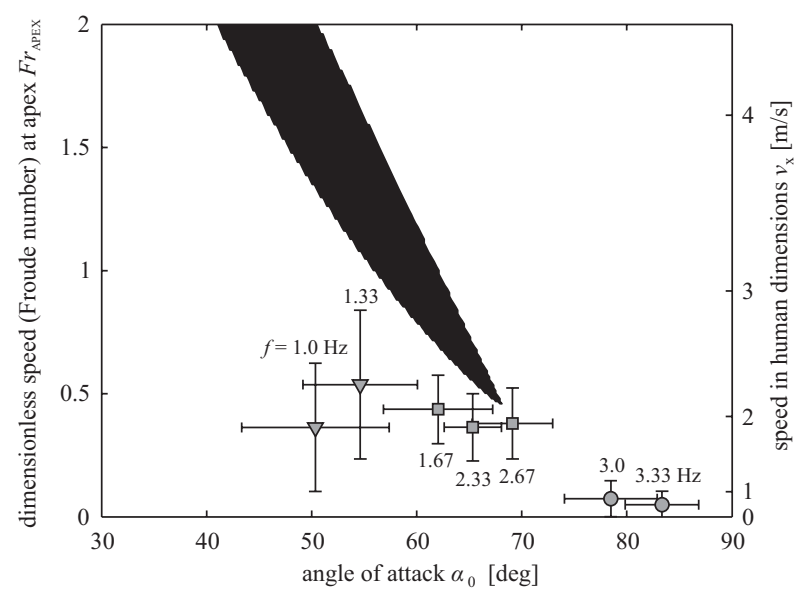

Fig. 7. Model prediction of angle of attack and dimensionless apex speed for self-stable running (black area, $\beta_{0}=150 \mathrm{deg}, \widetilde{c}=0.66$ ), and frequency dependent results (mean \pm s.d.) of JenaHopper hopping $\left(\theta_{0}=15 \mathrm{deg}\right)$. The symbol description is explained in Sec. III-C.

low speed, the Floquet multipliers indicate stable movements with few exceptions. The transition from forward hopping to hopping in place at $f=3.33 \mathrm{~Hz}$ is accompanied by a tendency toward instability.

Finally, we compare the robot experiments with the twosegment leg template. We detect the speed $v_{x}$ at the instant of apex and the angle of attack $\alpha_{0}$. Both variables are not directly controlled in the robot, but "occur" dependent on leg dynamics and hip oscillation. The nominal angle $\beta_{0}$ of the leg template is set to $150 \mathrm{deg}$. This is larger than that of the robot (132 deg) to simulate the extra nonlinearity in the leg force caused by the pretension of the robot's joint spring. Since the model and robot are of different sizes, we compare the resulting speeds using the dimensionless Froude number [14] given as $\mathrm{Fr}=v^{2} /\left(g l_{0}\right)$. Fig. 7 shows both the dimensionless speed and the angle of attack for some representative control settings and the model predicted region for self-stable running. Here, we identify three kinds of robot behavior. First, at medium frequencies (squares) the angle of attack and the velocity lie close to the lower end of the model predicted stability region. These hopping results were previously identified as stable solutions (Fig. 5(b)). The second region for low control frequencies (triangles) lies somewhat away from the model predicted area. Here, large ranges in velocity and leg angle $\alpha_{0}$ can be observed, which are a consequence of unstable locomotion. The third region with higher frequencies (circles) is found at high leg angles and low velocities. These outcomes are far away from the stable region of the two-segment leg, although they were identified as stable movements (Fig. 5(b)). The stable hopping solutions of the robot (squares and circles) cover the middle to lower-right region and extend the model prediction.

\section{Discussion AND CONCLUSION}

In this study, we proposed a conceptual model to discover the potential role of leg segmentation on running stability. Additionally, we conducted experiments on a robot testbed 

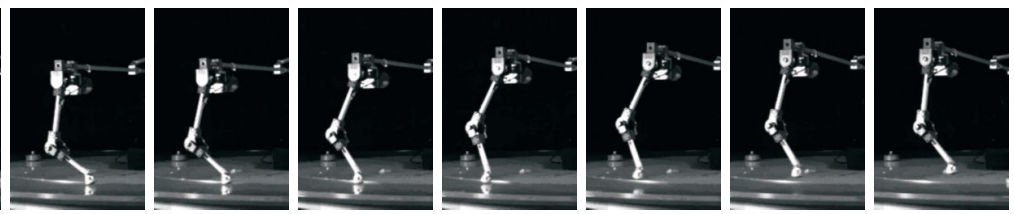

Fig. 8. Video sequence of JenaHopper hopping on a turntable. The direction of locomotion is from left to right.

to further understand the nature of elastic and segmented legs. In this section, we will discuss the model predictions and experimental results.

The two-segment model reveals that segmented legs provide self-stable running at an enlarged range of running speeds, i.e. at lower speeds, compared to prismatic legs with spring-like behavior. We found that in an almost straight leg configuration $\left(\beta_{0}=170 \mathrm{deg}\right)$, comparable to the knee joint in human legs, the minimum running speed is $1.1 \mathrm{~m} / \mathrm{s}$. This is much lower than the preferred gait transition speed between walking and running at about $2 \mathrm{~m} / \mathrm{s}$ [14]. Our robot experiments support the model prediction, showing stable locomotion at relatively low speeds (Fig. 6(b) and Fig. 7). A second model prediction and experimental outcome is that running becomes more robust to variations in the angle of attack. This suggests that even simple control strategies, e.g. swinging the leg back and forth, lead to stable periodic movements in systems with segmented legs. Robots with a similar leg design [15], [12] could take advantage of the mechanical self-stability identified in the model. However, a control of speed and apex height is possible by adapting the angle of attack [11].

Running animals and robots with spring-like leg behavior can stabilize running at different speeds by using simple leg strategies, e.g. adjustment of leg stiffness, adjustment of angle of attack, or a combination of both (Fig. 4(a) and [5]). In contrast, the two-segmented model indicates that an adjustment of joint stiffness to running speed is necessary (Fig. 4(b) and (c)), especially since a low or medium joint stiffness is preferred at low speeds. This model prediction is supported by human experiments where the knee joint stiffness increased with increasing running speed [16]. The required adjustment of joint stiffness to speed could be done by novel technical solutions, e.g. [17]. However, most robots, including JenaHopper, are restricted in speed due to motor performance [7].

The novel features of segmented legs with respect to running stability are consequences of the nonlinear relationship between leg length and leg force resulting in a decreasing leg stiffness with increasing leg compression (Fig. 3). The adaptation of leg stiffness, i.e. softening the leg spring, is a purely passive mechanism in segmented legs with springlike joint functions. This behavior allows for self-adapting the systems natural frequency to the induced hip oscillation.

All robot experiments result in lower velocities than predicted by the model (Fig. 7). This is probably due to the highly reduced nature of the model, i.e. it does not consider energy dissipation, the disabled pitch motion of the body, motor limitations and the pretension of the spring. Another finding in the experiments is a swing-leg retraction before touch-down (Fig. 6) which improves stability in spring-like legs [18]. Although, swing-leg retraction hardly influences stability in the two-segment model (unpublished results), it could gain stability in the robot with non-negligible leg mass: It prepares the leg for stance-leg retraction and, therefore, a reduction of impact forces and system perturbations is assumed. It remains for further studies on how leg mass influences running dynamics.

\section{REFERENCES}

[1] M. H. Raibert, Legged robots that balance. MIT Press, 1986.

[2] R. Blickhan, "The spring-mass model for running and hopping," $J$. Biomech., vol. 22, no. 11-12, pp. 1217-1227, 1989.

[3] T. A. McMahon and G. C. Cheng, "The mechanics of running: how does stiffness couple with speed?" J. Biomech., vol. 23, no. 1, pp. 65-78, 1990.

[4] H. Geyer, A. Seyfarth, and R. Blickhan, "Compliant leg behaviour explains basic dynamics of walking and running," Proc. $R$. Soc. London B, vol. 273, no. 1603, pp. 2861-2867, 2006.

[5] A. Seyfarth, H. Geyer, M. Guenther, and R. Blickhan, "A movement criterion for running," J. Biomech., vol. 35, no. 5, pp. 649-655, 2002.

[6] M. Ahmadi and M. Buehler, "The ARL monopod II running robot: Control and energetics," in Proc. IEEE Int. Conf. Robot. Autom., 1999, pp. 1689-1694.

[7] I. Poulakakis, J. A. Smith, and M. Buehler, "Modeling and experiments of untethered quadrupedal running with a bounding gait: The Scout II robot," Int. J. Robot. Res., vol. 24, no. 4, pp. 239-256, 2005.

[8] S. Kim, J. E. Clark, and M. R. Cutkosky, "iSprawl: Design and tuning for high-speed autonomous open-loop running," Int. J. Robot. Res., vol. 25, no. 9, pp. 903-912, 2006.

[9] F. Iida, J. Rummel, and A. Seyfarth, "Bipedal walking and running with compliant legs," in Proc. IEEE Int. Conf. Robot. Autom., 2007.

[10] R. J. Full and D. E. Koditschek, "Templates and anchors: neuromechanical hypotheses of legged locomotion on land," J. Exp. Biol., vol. 202, no. 23, pp. 3325-3332, 1999.

[11] Z. G. Zhang, Y. Fukuoka, and H. Kimura, "Stable quadrupedal running based on a spring-loaded two-segment legged model," in Proc. IEEE Int. Conf. Robot. Autom., 2004, pp. 2601-2606.

[12] F. Iida and R. Pfeifer, "Sensing through body dynamics," Robot. Auton. Syst., vol. 54, no. 8, pp. 631-640, 2006.

[13] W. J. Schwind and D. E. Koditschek, "Approximating the stance map of a 2-DOF monoped runner," J. Nonlinear Sci., vol. 10, no. 5, pp. $533-568,2000$.

[14] R. M. Alexander, "Bipedal animals, and their differences from humans," J. Anat., vol. 204, no. 5, pp. 321-330, 2004.

[15] L. R. Palmer, D. E. Orin, D. W. Marhefka, J. P. Schmiedeler, and K. J. Waldron, "Intelligent control of an experimental articulated leg for a galloping machine," in Proc. IEEE Int. Conf. Robot. Autom., 2003, pp. 3821-3827.

[16] J. Rummel, S. Lipfert, and A. Seyfarth, "Knee joint stiffness for selfstable running," J. Biomech., vol. 40, suppl. 2, p. S590, 2007.

[17] R. Van Ham, B. Vanderborght, M. Van Damme, B. Verrelst, and D. Lefeber, "MACCEPA: the actuator with adaptable compliance for dynamic walking bipeds," in Proc. Int. Conf. on Climbing and Walking Robots, 2005, pp. 759-766.

[18] A. Seyfarth, H. Geyer, and H. Herr, "Swing-leg retraction: a simple control model for stable running," J. Exp. Biol., vol. 206, no. 15, pp. 2547-2555., 2003. 